\title{
The incidence rate of corpus uteri cancer
} among females in Saudi Arabia: an observational
descriptive epidemiological analysis of data from
Saudi Cancer Registry 200I-2008

\author{
This article was published in the following Dove Press journal: \\ International Journal of Women's Health \\ 29 January 2014 \\ Number of times this article has been viewed
}

\author{
Ibrahim G Alghamdi' \\ Issam I Hussain' \\ Mohamed S Alghamdi² \\ Mohamed A El-Sheemy ${ }^{1,3}$ \\ 'University of Lincoln, Brayford Pool, \\ Lincoln, UK; ${ }^{2}$ Ministry of Health, \\ General Directorate of Health Affairs, \\ Al-Baha, Kingdom of Saudi Arabia; \\ ${ }^{3}$ Research and Development, Lincoln \\ Hospital, Lincolnshire Hospitals NHS \\ Trust, Lincoln, UK
}

Background: The present study reviews the epidemiological data on corpus uteri cancer among Saudi women, including its frequency, crude incidence rate, and age-standardized incidence rate (ASIR), adjusted by region and year of diagnosis.

Methods: A retrospective, descriptive epidemiological analysis was conducted of all the corpus uteri cancer cases recorded in the Saudi Cancer Registry between January 2001 and December 2008. The statistical analyses were performed using descriptive statistics, analysis of variance, Poisson regression, and a simple linear model.

Results: A total of 1,060 corpus uteri cancer cases were included. Women aged 60-74 years of age were most affected by the disease. The region of Riyadh in Saudi Arabia had the highest overall ASIR, at 4.4 cases per 100,000 female patients, followed by the eastern region, at 4.2, and Makkah, at 3.7. Jazan, Najran, and Qassim had the lowest average ASIRs, ranging from 0.8 to 1.4. A Poisson regression model using Jazan as the reference revealed that the corpus uteri cancer incidence rate ratio was significantly higher for the regions of Makkah, at 16.5 times (95\% confidence interval [CI]: 8.0-23.0), followed by Riyadh, at 16.0 times (95\% CI: 9.0-22.0), and the eastern region, at 9.9 times (95\% CI: 5.6-17.6). The northern region experienced the highest changes in ASIRs of corpus uteri cancer among female Saudi patients between 2001 and 2008.

Conclusion: There was a slight increase in the crude incidence rates and ASIRs for corpus uteri cancer in Saudi Arabia between 2001 and 2008. Older Saudi women were most affected by the disease. Riyadh, the eastern region, and Makkah had the highest overall disease ASIRs and incidence rate ratios, while Jazan, Najran, and Qassim had the lowest rates. Finally, the northern region experienced the greatest changes in ASIR during the studied period. Further analytical studies are necessary to determine potential risk factors of corpus uteri cancer among female Saudi patients. Keywords: cancer epidemiology, Saudi Cancer Registry, corpus uteri cancer, age standardized incidence rate

\section{Introduction}

Cancer of the corpus uteri has been reported to be the most common gynecologic malignancy in Europe and other countries, such as the United States of America, Russia, and Canada. ${ }^{1}$ The incidence of corpus uteri cancer is increased in countries with higher socioeconomic class $(5.5 \%)$ compared with that in countries with a lower socioeconomic class $(4.2 \%){ }^{2}$ In addition, it has been reported that the incidence of corpus uteri cancer increases with age and life expectancy. ${ }^{3}$ In the United States and Europe, corpus uteri cancer is the fourth most common cancer in women, after colorectal, breast, and lung cancer. ${ }^{2,4}$ It accounts for approximately $6 \%$ of all cancers, with
Correspondence: Ibrahim G Alghamdi University of Lincoln, School of Life Sciences, Brayford Pool, Lincoln, LN6 7TS, UK

Tel +44| I6 276 I913

Email bio-stat@hotmail.com 
49,560 diagnosed cases and up to 8,190 deaths annually. ${ }^{4}$ The highest age-standardized incidence rate (ASIR) of the disease is observed in North America, at 18 cases per 100,000 women, with the rate being 15 per 100,000 women in Canada. ${ }^{5}$ Additionally, Eastern European countries are the most affected by corpus uteri cancer, whereas Africa, and southern and eastern Asia are the least affected. ${ }^{5}$

In Saudi Arabia, epidemiological trends observed from the Saudi Cancer Registry (SCR) between 2001 and $2008^{6}$ offer more details on the disease pattern in different regions of the country. In 2008, corpus uteri cancer was reported as one of the most common types of cancer in Saudi Arabia; its incidence in women was the sixth highest as compared with the incidence of all other types of cancer. The International Agency for Research on Cancer estimated that in 2008, the ASIR for corpus uteri cancer was 4.4 cases per 100,000 female patients, and the age-standardized mortality rate was 1.2 per 100,000 female patients. ${ }^{7}$ Furthermore, the registry of the King Faisal Specialist Hospital and Research Centre (2011) recorded 1,048 cases of corpus uteri cancer admitted to the hospital from 1975 to $2011 .^{8}$ The ASIR of corpus uteri cancer in Saudi Arabia is slightly lower than that in some other Arabian Gulf countries. Specifically, in 2008, the reported ASIRs for Bahrain, Qatar, and the United Arab Emirates were 6.1, 4.5, and 4.5, respectively. ${ }^{7}$ This study aimed to investigate and describe the crude incidence rate (CIR) and ASIR of corpus uteri cancer among women in Saudi Arabia, by conducting an observational, descriptive epidemiological analysis of the data from the SCR between 2001 and 2008, as well as to examine the disease distribution, based on patients' characteristics, location, and time of diagnosis. ${ }^{9}$

\section{Materials and methods}

We conducted a retrospective, descriptive epidemiological study of all corpus uteri cancer cases diagnosed between January 2001 and December 2008 that were recorded in the SCR. The SCR is a publicly available and easily accessible population-based registry, established in 1994 by the Saudi Arabian Ministry of Health. Therefore, no ethical approval was required for the observational descriptive epidemiological studies. However, the data could not be obtained directly from the SCR; we instead obtained the data via its published reports. There were no available data from 1994 to 2000, and the latest SCR published report was in 2008 .

Since 2001, the SCR has provided reports on the patterns of cancer incidence in Saudi Arabia, with a primary objective of defining the population-based incidence of the disease. Based on these data, comprehensive reports for 13 administrative regions are currently available from 2001 to 2008, and they discuss the frequency, with the percentage of cases, CIR, and ASIR, adjusted by the provinces of Saudi Arabia, age group, and the year of diagnosis. The present study was conducted using such reports to critically gather all information from the SCR, to investigate the descriptive epidemiology of corpus uteri cancer among Saudi Arabian women.

For the data analysis, we used the Statistical Package for Social Sciences version 20.0. (IBM Corporation, Armonk, NY, USA). A descriptive analysis of the epidemiological data was performed by calculating the overall percentage, CIR, and ASIR, adjusted by age group, region, and year of diagnosis. The percentage was calculated by dividing the number of corpus uteri cancer cases by the total number of cancer cases in women. Furthermore, an analysis of variance was performed to determine any significant differences among the regions and years of diagnosis. In addition, a linear regression model examining the strength of the association between independent variables and an outcome was applied to predict the annual CIR and ASIR for corpus uteri cancer among Saudi women. ${ }^{10}$ A Poisson regression model was also employed to calculate the incidence rate ratio (IRR) of corpus uteri cancer cases in each region of Saudi Arabia. The overall age-standardized rates per 100,000 female patients were calculated for all SCR reports, based on a (Segi) standard for world population with an intermediate age (Table 1). The

Table I Segi* (world) standard for making comparisons with an intermediate-age population

\begin{tabular}{ll}
\hline Age group, years & Segi (world) standard \\
\hline $0-4$ & 12,000 \\
$5-9$ & 10,000 \\
$10-14$ & 9,000 \\
$15-19$ & 9,000 \\
$20-24$ & 8,000 \\
$25-29$ & 8,000 \\
$30-34$ & 6,000 \\
$35-39$ & 6,000 \\
$40-44$ & 6,000 \\
$45-49$ & 6,000 \\
$50-54$ & 5,000 \\
$55-59$ & 4,000 \\
$60-64$ & 4,000 \\
$65-69$ & 3,000 \\
$70-74$ & 2,000 \\
$75+$ & 2,000 \\
Total & 100,000
\end{tabular}

Note: *The Segi world standard can be used in a direct method of standardization to calculate the ASIR and ASMR. ${ }^{14}$

Abbreviations: ASIR, age-standardized incidence rate; ASMR, age-standardized mortality rate. 
ASIR is particularly important when comparing different populations with respect to age. ${ }^{11,12}$

\section{Results}

A total of 1,060 corpus uteri cancer cases were recorded in the SCR between January 2001 and December 2008. The number of corpus uteri cancer cases increased slightly from 2001 to 2008 . Figure $1 \mathrm{~A}$ and $1 \mathrm{~B}$ show that there were 87 cases in $2001(2.8 \%$; 95\% confidence interval [CI]: $1.9 \%-3.7 \%)$. This number increased to 143 by 2005 , representing an increase of $0.8 \%$, and decreased to $140(0.3 \%)$ by 2006 . By the end of 2008, the number of corpus uteri cancer cases was 194 (4.5\%), the highest reported by the SCR (2008).

The average number and percentage of corpus uteri cancer cases between 2001 and 2008, adjusted by age group, were calculated from the SCR data (Table 2 and Figure 1C and 1D). The age groups reported were 0-14, 15-29, 30-44, 45-59, $60-74$, and $>75$ years. According to the overall numbers and percentages from 2001 to 2008, the age group most affected by corpus uteri cancer included older women aged 60-74
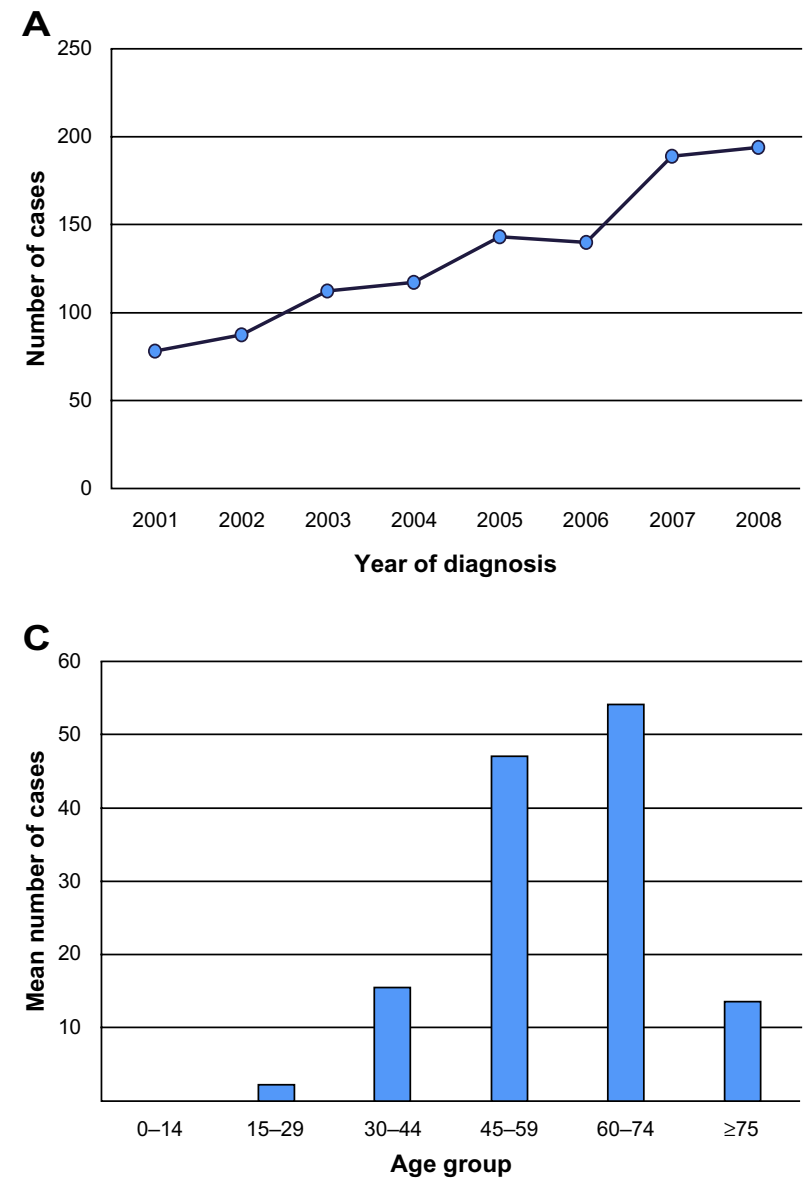

years, with an average of 54 cases, representing $40 \%$ of all the corpus uteri cancer cases. The second most affected age group were those aged 45-59 years, with 47 cases (35\%). The younger groups of female patients aged 0-14 and 15-29 years recorded the lowest overall numbers and percentages, with less than 15 cases per group (1.5\% and $12 \%$, respectively, of all the corpus uteri cancer cases).

The CIR, adjusted by the year of diagnosis, indicated a steady increase in the number of corpus uteri cancer cases among women in Saudi Arabia. According to Table 3 and Figure 2A, a CIR of 1.0 case (95\% CI: 0.7-1.3) per 100,000 women was estimated in 2001, whereas in 2007 and 2008, the estimated CIR was 2.2 cases (95\% CI: 1.8-2.6) per 100,000 women. This CIR was significantly higher than that of all the other years of diagnosis $(F[7,96]=4.280, P<0.05)$. A linear regression equation indicated that the annual increase in the CIR for corpus uteri cancer among Saudi women could be predicted by the formula $0.6+(0.15 \times$ years $)$. Thus, the CIR of corpus uteri cancer may have increased by an average of 0.15 per 100,000 Saudi women per year.

B

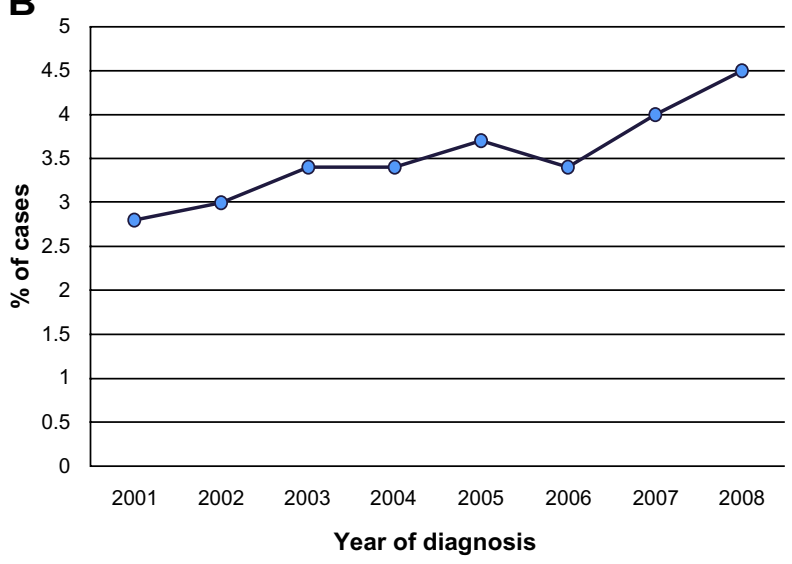

D

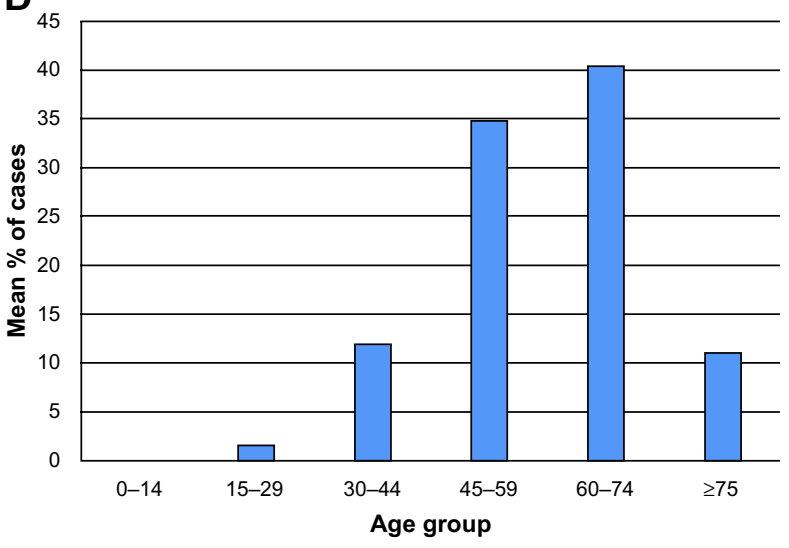

Figure I Number and percentage of corpus uteri cancer cases in Saudi Arabia from $200 \mathrm{I}$ to 2008 and overall number and percentage of corpus uteri cancer cases, distributed by age group, in Saudi Arabia from 2001 to 2008.

Notes: (A) Number of corpus uteri cancer cases in Saudi Arabia from $200 \mathrm{I}$ to 2008. (B) Percentage of corpus uteri cancer cases in Saudi Arabia from $200 \mathrm{I}$ to 2008. (C) Overall number of corpus uteri cancer cases, distributed by age group, in Saudi Arabia from 200I to 2008. (D) Overall percentage of corpus uteri cancer cases, distributed by age group, in Saudi Arabia from 2001 to 2008. 
Table 2 Corpus uteri cancer cases, distributed by age group, in Saudi Arabia, from 200I to 2008

\begin{tabular}{|c|c|c|c|c|c|c|c|c|c|c|c|c|c|c|}
\hline \multirow[t]{3}{*}{ Year } & \multicolumn{14}{|c|}{ Age group of corpus uteri cancer cases } \\
\hline & \multicolumn{2}{|c|}{$0-14$} & \multicolumn{2}{|c|}{ I 5-29 } & \multicolumn{2}{|c|}{$30-44$} & \multicolumn{2}{|c|}{ 45-59 } & \multicolumn{2}{|c|}{$60-74$} & \multicolumn{2}{|c|}{$\geq 75$} & \multicolumn{2}{|l|}{ Total } \\
\hline & No & $\%$ & No & $\%$ & No & $\%$ & No & $\%$ & No & $\%$ & No & $\%$ & Total & $\%$ of total \\
\hline 2001 & I & I & 2 & 2 & 10 & 13 & 26 & 33 & 22 & 28 & 17 & 22 & 78 & 2.8 \\
\hline 2002 & 0 & 0 & 2 & 2 & 8 & 9 & 32 & 37 & 37 & 43 & 8 & 9 & 87 & 3.0 \\
\hline 2003 & 0 & 0 & 0 & 0 & 17 & 15 & 31 & 28 & 52 & 46 & 12 & II & 112 & 3.4 \\
\hline 2004 & 0 & 0 & 2 & 2 & 16 & 14 & 33 & 28 & 52 & 44 & 14 & 12 & 117 & 3.4 \\
\hline 2005 & 0 & 0 & 2 & I & 19 & 13 & 54 & 38 & 56 & 39 & 12 & 8 & 143 & 3.7 \\
\hline 2006 & 0 & 0 & 3 & 2 & 17 & 12 & 45 & 32 & 61 & 44 & 13 & 9 & 140 & 3.4 \\
\hline 2007 & 0 & 0 & 2 & 1 & 20 & II & 80 & 42 & 74 & 39 & 13 & 7 & 189 & 4.0 \\
\hline 2008 & 0 & 0 & 4 & 2 & 16 & 8 & 78 & 40 & 77 & 40 & 19 & 10 & 194 & 4.5 \\
\hline Average & 0.1 & 0.1 & 2.1 & 1.5 & 15 & 12 & 47 & 35 & 54 & 40 & 14 & II & 132.5 & 3.5 \\
\hline
\end{tabular}

The overall CIR of corpus uteri cancer from 2001 to 2008 , adjusted by the region of Saudi Arabia, was also calculated from the SCR data, as shown in Table 4 and Figure 2B. The region of Makkah had the highest overall CIR for corpus uteri cancer, at 2.0 cases (95\% CI: 1.4-2.6) per 100,000 female patients, followed by Riyadh, at 1.9 (95\% CI: 1.7-2.2), and the eastern region, at 1.8 (95\% CI: 1.3-2.2). The overall CIRs of corpus uteri cancer were significantly higher in these regions than in the regions of Jazan, Najran, and Qassim $(F[12,91]=5.212, P<0.001)$. Furthermore, the regions of Jazan, Najran, and Qassim had the lowest average CIRs for corpus uteri cancer among Saudi women, at 0.4 (95\% CI: $0.1-0.7), 0.5$ (95\% CI: 0.1-0.9), and 0.7 (95\% CI: 0.5-0.9), respectively.

Similarly, the ASIR of corpus uteri cancer, adjusted by the year of diagnosis, was calculated from the SCR data (Table 3 and Figure 2C). There was a slight increase in the number of diagnoses between 2001 and 2008, with the highest ASIR, of 4.0 cases per 100,000 women, recorded in 2007 and 2008 (95\% CI: 2.2-4.0). The linear regression

Table 3 Confidence intervals for the \%, CIR, and ASIR of corpus uteri cancer cases in Saudi Arabia, from 200I to 2008

\begin{tabular}{|c|c|c|c|c|c|c|}
\hline \multirow[t]{2}{*}{ Year } & \multicolumn{2}{|l|}{$\%$} & \multicolumn{2}{|c|}{$\begin{array}{l}\text { CIR per } \\
100,000 \text { females }\end{array}$} & \multicolumn{2}{|c|}{$\begin{array}{l}\text { ASIR per } \\
100,000 \text { females }\end{array}$} \\
\hline & $\%$ & $95 \% \mathrm{Cl}$ & CIR & $95 \% \mathrm{Cl}$ & ASIR & $95 \% \mathrm{Cl}$ \\
\hline 2001 & 2.8 & $1.9-3.7$ & 1.0 & $0.7-1.3$ & 2.0 & I.2-2.8 \\
\hline 2002 & 3.0 & $2.1-3.9$ & I.I & $0.8-1.4$ & 2.2 & $1.1-3.3$ \\
\hline 2003 & 3.4 & $2.0-4.8$ & 1.4 & $0.9-1.9$ & 2.8 & $1.6-4.0$ \\
\hline 2004 & 3.4 & $2.4-4.4$ & 1.4 & $1.0-1.8$ & 2.8 & $1.9-3.7$ \\
\hline 2005 & 3.7 & $2.5-4.9$ & 1.7 & I.3-2.I & 3.4 & $2.5-4.3$ \\
\hline 2006 & 3.4 & $2.6-4.2$ & 1.6 & $1.2-2.0$ & 3.6 & $2.9-4.3$ \\
\hline 2007 & 4.0 & $3.1-4.9$ & 2.2 & I.7-2.7 & 4.0 & $2.9-5.1$ \\
\hline 2008 & 4.5 & $3.5-5.5$ & 2.2 & I.8-2.6 & 4.0 & $3.2-4.8$ \\
\hline Overall & 4.0 & $3.0-4.8$ & 2.0 & I.5-2.5 & 3.0 & $2.0-3.8$ \\
\hline
\end{tabular}

Abbreviations: ASIR, age-standardized incidence rate; CIR, crude incidence rate; $\mathrm{Cl}$, confidence interval. equation to predict the annual ASIR of corpus uteri cancer among Saudi Arabian women was calculated to be $1.6+$ ( $0.22 \times$ years). Accordingly, the ASIR of corpus uteri cancer increased by a predicted average of 0.22 per 100,000 female Saudi patients per year. Similarly, as shown in Table 4 and Figure 2D, the region of Riyadh had the highest overall ASIR for the disease, at 4.4 cases (95\% CI: 3.9-4.9) per 100,000 women, followed by the eastern region, at 4.2 (95\% CI: 3.4-5.0), and Makkah, at 3.7 (95\% CI: 2.7-4.7). The overall ASIRs of corpus uteri cancer were significantly higher in these regions than in the regions of Jazan, Najran, and $\operatorname{Qassim}(F[12,91]=6.907, P<0.001)$. Alternatively, the regions of Jazan, Najran, and Qassim had the lowest overall ASIRs, at 0.8 (95\% CI: 0.3-1.3), 1.0 (95\% CI: 0.3-1.8), and 1.4 (95\% CI: 0.7-2.1), respectively.

A Poisson regression model was used to calculate the IRR of corpus uteri cancer cases in the different regions of Saudi Arabia. The model was statistically significant (likelihood ratio [LR] $\left.\chi^{2}(13)=1,918.9 ; P<0.001\right)$. Jazan was used as the reference region for comparison with other provinces of Saudi Arabia. As shown in Table 5, the IRRs were significantly higher $(P<0.001)$ for the regions of Makkah, at 16.5 times (95\% CI: 8.0-23.0), Riyadh, at 16 times (95\% CI: 9.0-22.0), and the eastern region, at 9.9 times (95\% CI: 5.6-17.6). Furthermore, the IRRs of corpus uteri cancer were also slightly higher $(P<0.001)$ for both Asir and Madinah, at 3.6 times (95\% CI: 1.9-6.6) and 3.5 times (95\% CI: 1.9-6.5), respectively.

Finally, the differences in CIR and ASIR between 2001 and 2008 were calculated from the SCR reports. The pattern of corpus uteri cancer incidence among Saudi women in different regions is shown in Table 6. The greatest change in ASIR was observed in the northern region, at 5.0 per 100,000 women. However, the CIR and ASIR for all regions, except for the values for the northern region, 

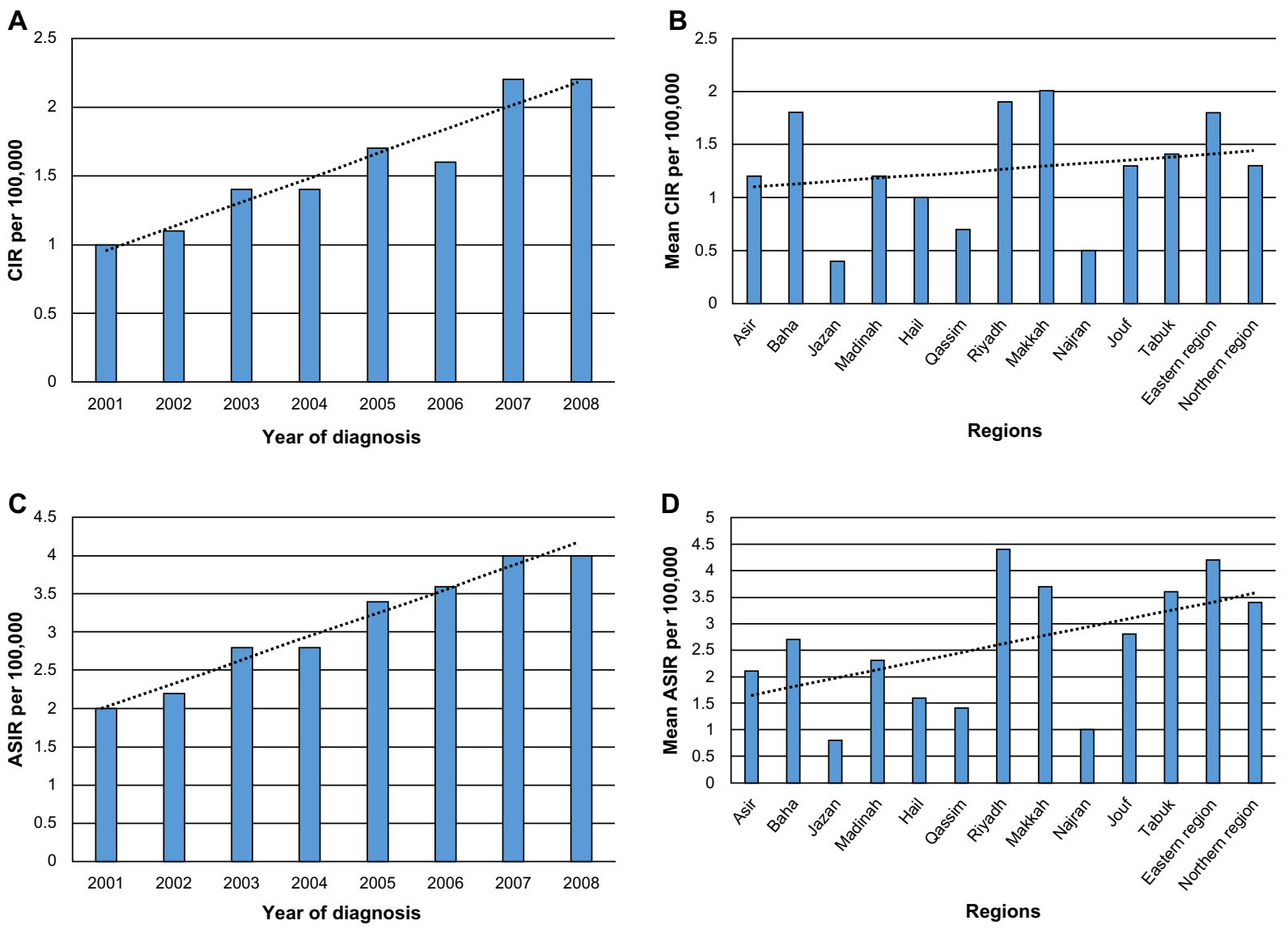

Figure 2 (A) The CIR of corpus uteri cancer cases among females in Saudi Arabia from 2001 to 2008. (B) Overall CIR of corpus uteri cancer cases, distributed by region, in Saudi Arabia from $200 \mathrm{I}$ to 2008. (C) The ASIR of corpus uteri cancer cases among females in Saudi Arabia from 200I to 2008. (D) Overall ASIR of corpus uteri cancer cases, distributed by region, in Saudi Arabia from $200 \mathrm{I}$ to 2008.

Abbreviations: ASIR, age-standardized incidence rate; CIR, crude incidence rate.

Table 4 Confidence interval for the overall \%, CIR, and ASIR of corpus uteri cancer cases in the regions of Saudi Arabia, from 200 I to 2008

\begin{tabular}{|c|c|c|c|c|c|c|}
\hline \multirow[t]{2}{*}{ Regions } & \multicolumn{2}{|c|}{ Overall \% } & \multicolumn{2}{|c|}{$\begin{array}{l}\text { Overall CIR } \\
\text { per } 100,000 \\
\text { females }\end{array}$} & \multicolumn{2}{|c|}{$\begin{array}{l}\text { Overall ASIR } \\
\text { per } 100,000 \\
\text { females }\end{array}$} \\
\hline & $\%$ & $95 \% \mathrm{Cl}$ & CIR & $95 \% \mathrm{Cl}$ & ASIR & $95 \% \mathrm{Cl}$ \\
\hline Asir & 3.4 & $2.3-4.4$ & 1.2 & $0.8-1.6$ & 2.1 & I.4-2.7 \\
\hline Baha & 5.0 & $3.7-6.8$ & 1.8 & I. $2-2.3$ & 2.7 & $1.9-3.4$ \\
\hline Jazan & 1.7 & $0.3-3.1$ & 0.4 & $0.1-0.7$ & 0.8 & $0.3-1.3$ \\
\hline Madinah & 3.2 & $2.0-4.3$ & 1.2 & $0.6-1.9$ & 2.3 & I.2-3.5 \\
\hline Hail & 3.3 & I.5-5. I & 1.0 & $0.4-1.7$ & 1.6 & $0.7-2.5$ \\
\hline Qassim & 1.9 & I.3-2.5 & 0.7 & $0.5-0.9$ & 1.4 & $0.7-2.1$ \\
\hline Riyadh & 3.5 & $3.2-3.9$ & 1.9 & I.7-2.2 & 4.4 & $3.9-4.9$ \\
\hline Makkah & 4.1 & $3.5-4.8$ & 2.0 & $1.4-2.6$ & 3.7 & $2.7-4.7$ \\
\hline Najran & 1.8 & $0.6-2.9$ & 0.5 & $0.1-0.9$ & 1.0 & $0.3-1.8$ \\
\hline Jouf & 3.5 & I.I-5.9 & 1.3 & $0.4-2.1$ & 2.8 & $0.7-4.8$ \\
\hline Tabuk & 3.7 & $2.3-5.0$ & I.4 & $0.8-1.4$ & 3.6 & $2.1-5.0$ \\
\hline $\begin{array}{l}\text { Eastern } \\
\text { region }\end{array}$ & 3.4 & $2.8-3.9$ & 1.8 & I.3-2.2 & 4.2 & $3.4-5.0$ \\
\hline $\begin{array}{l}\text { Northern } \\
\text { region }\end{array}$ & 4.0 & $2.5-5.5$ & 1.3 & $0.8-1.8$ & 3.4 & $2.0-4.8$ \\
\hline
\end{tabular}

Abbreviations: ASIR, age-standardized incidence rate; CIR, crude incidence rate; $\mathrm{Cl}$, confidence interval. remained below the predicted values by the linear regression analyses (CIR: $0.6+[0.15 \times 8$ years $]=1.8$ cases per 100,000 females; ASIR: $1.6+[0.22 \times 8$ years $]=3.4$ per 100,000 females).

\section{Discussion}

It is important to monitor and update the incidence rate of corpus uteri cancer among Saudi women in all regions of Saudi Arabia. In the present study, we focused on the CIR and ASIR of corpus uteri cancer in Saudi Arabia from 2001 to 2008. Notably, to the best of our knowledge, this is the first descriptive epidemiology study of corpus uteri cancer among women in different regions of Saudi Arabia. It therefore provides an important explanation of the observed trends, confirming the significance of the disease.

Our results indicate that Riyadh, Makkah, and the eastern region have the highest overall ASIRs for corpus uteri cancer among women in Saudi Arabia. However, the overall ASIRs of these regions did not increase above 
Table 5 Poisson regression model calculation of the IRR

\begin{tabular}{llll}
\hline Regions & IRR & P-value & $\mathbf{9 5 \% ~ C I}$ \\
\hline I. Asir & 3.6 & $0.00 I^{*}$ & $1.9-6.6$ \\
2. Baha & I.4 & 0.23 & $0.8-2.0$ \\
3. Jazan & I Reference & & \\
4. Madinah & 3.5 & 0.00 I* $^{*}$ & $1.9-6.5$ \\
5. Hail & I.I & 0.3 & $0.6-2.2$ \\
6. Qassim & 1.3 & 0.38 & $0.7-2.5$ \\
7. Riyadh & 16 & $0.00 I^{*}$ & $9.0-22.0$ \\
8. Makkah & 16.5 & $0.00 I^{*}$ & $8.0-23.0$ \\
9. Najran & 0.4 & 0.06 & $0.2-0.9$ \\
I0. Jouf & 0.9 & 0.76 & $0.4-1.9$ \\
II. Tabuk & 1.7 & 0.09 & $0.9-3.3$ \\
I2. Eastern region & 9.9 & $0.00 I^{*}$ & $5.6-17.6$ \\
I3. Northern region & 0.8 & 0.06 & $0.4-1.6$ \\
\hline
\end{tabular}

Notes: The Poisson regression model was used to verify whether there was an effect due to geographical area and year of diagnosis. *Significant.

Abbreviations: $\mathrm{Cl}$, confidence interval; IRR, incidence rate ratio.

the expected levels from 2001 to 2008. Alternatively, the regions of Jazan, Najran, and Qassim had the lowest overall ASIRs of corpus uteri cancer among women in Saudi Arabia, between 2001 and 2008. In addition, the IRRs of corpus uteri cancer were significantly higher in Makkah, at 16.5 times, followed by Riyadh, at 16 times, and the eastern region, at 9.9 times than that in the reference region of Jazan. However, the increasing number of cases were dependent on the region. For instance, regions such as Jazan, Najran, and Qassim exhibited the lowest rates of increase compared with Makkah, Riyadh, and the eastern region. Such regional differences might be due to the socioeconomic status, environmental factors, and lifestyle habits.
Socioeconomic status, improvement in technology, and awareness among women may influence the burden of cancer and its incidence rate. The more technologically developed and affluent areas exhibited a higher incidence of corpus uteri cancer. This suggests that people in higher socioeconomic areas receive treatment more often, resulting in a greater number of cases being diagnosed and reported than that in areas with lower socioeconomic status, where the numbers may be underestimated due to undetected cases. However, we suspect that the false impact of socioeconomic status on incidence rate of cancer is only observed when the period of study is short (months). While, with longer surveyed time (years), all cases should eventually be detected resulting in a more accurate reported incidence rate. The period of time from case diagnosis may be considered a potential bias when studying the real effect of socioeconomic status on cancer. However, it is challenging to study the relationship between socioeconomic status and the incidence rate of corpus uteri cancer because the SCR does not collect data on socioeconomic status for the different regions in Saudi Arabia.

In the present study, we observed that cancer of the corpus uteri occurred more often among older women aged 60-74 years, whereas lower numbers of cases were reported among women aged 44 years and younger. Based on this result, reproductive factors, which have been linked with increased exposure to endogenous estrogens, may influence the risks for the disease. Other explanations in the age groups most affected by corpus uteri cancer include early menarche, late menopause, or family history. ${ }^{12,13}$ In our findings, we

Table 6 The differences in the \%, CIR, and ASIR of corpus uteri cancer cases in the regions of Saudi Arabia, between $200 \mathrm{I}$ and 2008

\begin{tabular}{|c|c|c|c|c|c|c|c|c|c|c|c|c|}
\hline \multirow[t]{2}{*}{ Regions } & \multicolumn{4}{|l|}{$\%$} & \multicolumn{4}{|c|}{ CIR per 100,000 females } & \multicolumn{4}{|c|}{ ASIR per 100,000 females } \\
\hline & 2008 & 2001 & Difference & $P$-value & 2008 & 2001 & Difference & $\overline{P \text {-value }}$ & 2008 & 2001 & Difference & $P$-value \\
\hline Asir & 3.8 & 1.0 & 2.8 & 0.20 & 1.6 & 0.3 & 1.3 & 0.34 & 2.4 & 0.4 & 2.0 & 0.23 \\
\hline Baha & 5.9 & 2.5 & 3.4 & 0.24 & 2.2 & 0.4 & 1.8 & 0.26 & 3.0 & 0.8 & 2.2 & 0.25 \\
\hline Jazan & 5.4 & 0.8 & 4.6 & 0.06 & 1.5 & 0.2 & 1.3 & 0.31 & 2.8 & 0.3 & 2.5 & 0.13 \\
\hline Madinah & 4.0 & 2.2 & 1.8 & 0.48 & 1.8 & 0.6 & 1.2 & 0.44 & 3.0 & 1.5 & 1.5 & 0.48 \\
\hline Hail & 7.8 & 3.4 & 4.4 & 0.19 & 2.8 & 0.8 & 1.0 & 0.61 & 3.8 & 1.5 & 2.3 & 0.07 \\
\hline Qassim & 1.6 & 1.4 & 0.2 & 0.91 & 0.7 & 0.7 & 0.0 & 1.0 & I.I & 1.0 & 0.1 & 0.49 \\
\hline Riyadh & 3.4 & 2.9 & 0.5 & 0.84 & 2.1 & 1.5 & 0.6 & 0.75 & 4.3 & 3.7 & 0.6 & 0.83 \\
\hline Makkah & 5.2 & 4.4 & 0.8 & 0.81 & 2.9 & 1.4 & 1.5 & 0.47 & 5.2 & 2.7 & 2.6 & 0.35 \\
\hline Najran & 4.5 & 0.0 & 4.5 & 0.06 & 1.5 & 0.0 & 1.5 & 0.21 & 2.7 & 0.0 & 2.7 & 0.10 \\
\hline Jouf & 1.6 & 0.0 & 1.6 & 0.21 & 0.6 & 0.0 & 0.6 & 0.44 & I.I & 0.0 & I.I & 0.27 \\
\hline Tabuk & 4.7 & 1.2 & 3.5 & 0.14 & 1.9 & 0.4 & 1.5 & 0.31 & 3.6 & 1.5 & 1.8 & 0.42 \\
\hline $\begin{array}{l}\text { Eastern } \\
\text { region }\end{array}$ & 4.0 & 3.2 & 0.8 & 0.77 & 2.4 & 1.4 & 1.0 & 0.61 & 5.4 & 3.5 & 1.9 & 0.52 \\
\hline $\begin{array}{l}\text { Northern } \\
\text { region }\end{array}$ & 3.7 & 0.0 & 3.7 & $0.05^{*}$ & 1.5 & 0.0 & 1.5 & 0.21 & 5.1 & 0.0 & 5.1 & $0.02 *$ \\
\hline
\end{tabular}

Note: *Significant.

Abbreviations: ASIR, age-standardized incidence rate; CIR, crude incidence rate. 
have analyzed the changes in percentage, CIR, and ASIR of corpus uteri cancer among women in different regions of Saudi Arabia, from 2001 to 2008. The northern region had the highest differences in the ASIR of corpus uteri cancer during the studied period, with changes above the predicted values of rates, between 2001 and 2008. However, the likely explanation for these differences in rates is that the northern region was affected more by corpus uteri cancer over an 8-year period (2001-2008).

Despite a lack of data on the geographic distribution of corpus uteri cancer among women in Saudi Arabia, except for data from the SCR reports, we aimed to determine the actual pattern and trends for corpus uteri cancer among Saudi women in different regions, from 2001 to 2008. Our findings are significant to other researchers and decision makers in Saudi Arabia as our study provides epidemiological descriptive results of corpus uteri cancer among women in different provinces of the country. Therefore, these results will contribute positively and may help in developing hypotheses on the potential risk factors of corpus uteri cancer in the most affected regions of Saudi Arabia, as well as further analytic epidemiologic studies to identify the association between exposures and disease.

\section{Conclusion}

Our study revealed that the CIRs and ASIRs of corpus uteri cancer among Saudi women have increased slightly. Riyadh, the eastern region, and Makkah had the highest overall CIRs and ASIRs in Saudi Arabia, whereas Jazan, Najran, and Qassim recorded the lowest rates. The results of a Poisson regression model for IRR of corpus uteri cancer were concerning in certain regions, such as Makkah, Riyadh, and the eastern region. As mentioned earlier, the northern region reported the greatest differences in the ASIR of corpus uteri cancer among Saudi women from
2001 to 2008. Older Saudi women were most affected by corpus uteri cancer. Further analytical studies are necessary to investigate the potential risk factors of corpus uteri cancer among Saudi women.

\section{Disclosure}

The authors report no conflicts of interest in this work.

\section{References}

1. Bray F, Loos AH, Oostindier M, Weiderpass E. Geographic and temporal variations in cancer of the corpus uteri: incidence and mortality in pre- and postmenopausal women in Europe. Int J Cancer. 2005;117(1): $123-131$.

2. Jemal A, Bray F, Center MM, Ferlay J, Ward E, Forman D. Global cancer statistics. CA Cancer J Clin. 2011;61(2):69-90.

3. Cust AE. Physical activity and gynecologic cancer prevention. Recent Results Cancer Res. 2011;186:159-185.

4. American Cancer Society. Cancer Facts and Figures 2013. Atlanta, GA: American Cancer Society; 2013.

5. Parkin DM, Bray F, Ferlay J, Pisani P. Global cancer statistics, 2002 CA Cancer J Clin. 2005;55(2):74-108.

6. Saudi Cancer Registry. Cancer Incidence Report. Saudi Arabia. Riyadh: Ministry of Health; 2008. Available from http://www.oncology.org.sa/. Accesse October 5, 2013.

7. International Agency for Research on Cancer. GLOBOCAN. Cancer Fact Sheet. International Agency for Research on Cancer; 2008. Available from http://globocan.iarc.fr/factsheets/cancers/all.asp. Accessed October 10, 2013.

8. King Faisal Specialist Hospital and Research Centre. Tumor Registry. Annual Report. Riyadh: King Faisal Specialist Hospital and Research Centre; 2011.

9. Friis RH, Sellers TA. Epidemiology for Public Health Practice. 4th ed. Sudbury, MA: Jones \& Bartlett; 2009.

10. Tabatabai MA, Eby WM, Li H, Bae S, Singh KP. TELBS robust linear regression method. Open Access Med Stat. 2012;2012(2):65-84.

11. Ahmad OB, Boschi-Pinto C, Lopez AD, Murray CJL, Lozano R Inoue. Age Standardization of Rates: A New WHO Standard. GPE Discussion Paper Series: No 31. Geneva: EIP/GPE/EBD World Health Organization; 2001.

12. Doll R, Payne PM, Waterhouse JAH, editors. Cancer Incidence in Five Countries, Vol 1. Berlin: Springer-Verlag; 1966.

13. Zambon P, La Rosa F. Gynecological cancers: cervix, corpus uteri, ovary Epidemiol Prev. 2004;28(Suppl 2):S68-S74. English and Italian.

14. Segi M. Cancer mortality for selected sites in 24 countries (1950-57) Department of Public Health, Tohoku University of Medicine, Sendai, Japan. 1960
International Journal of Women's Health

\section{Publish your work in this journal}

The International Journal of Women's Health is an international, peerreviewed open-access journal publishing original research, reports, editorials, reviews and commentaries on all aspects of women's healthcare including gynecology, obstetrics, and breast cancer. The manuscript management system is completely online and includes

\section{Dovepress}

a very quick and fair peer-review system, which is all easy to use. Visit http://www.dovepress.com/testimonials.php to read real quotes from published authors. 